\title{
The DPC4/SMAD4 genetic status determines recurrence patterns and treatment outcomes in resected pancreatic ductal adenocarcinoma: A prospective cohort study
}

\author{
Sang Hyun Shin ${ }^{1, *}$, Hwa Jung Kim ${ }^{2, *}$, Dae Wook Hwang ${ }^{1}$, Jae Hoon Lee ${ }^{1}$, Ki Byung \\ Song ${ }^{1}$, Eunsung Junn, In Kyong Shim ${ }^{4}$, Seung-Mo Hong ${ }^{5}$, Hyoung Jung Kim ${ }^{6}$, \\ Kwang-Min Park ${ }^{1}$, Young-Joo Lee ${ }^{1}$, Song Cheol Kim ${ }^{1}$ \\ ${ }^{1}$ Division of Hepato-Biliary and Pancreatic Surgery, Department of Surgery, Asan Medical Center, University of Ulsan College \\ of Medicine, Seoul, South Korea \\ ${ }^{2}$ Department of Preventive Medicine, University of Ulsan College of Medicine, Seoul, South Korea \\ ${ }^{3}$ Department of Biomedical Sciences, University of Ulsan College of Medicine, Seoul, South Korea \\ ${ }^{4}$ Biomedical Research Center, Asan Institute for Life Sciences, Asan Medical Center, Seoul, South Korea \\ ${ }^{5}$ Department of Pathology, Asan Medical Center, University of Ulsan College of Medicine, Seoul, South Korea \\ ${ }^{6}$ Department of Radiology and the Research Institute of Radiology, Asan Medical Center, University of Ulsan College of \\ Medicine, Seoul, South Korea \\ "These authors have contributed equally to this work \\ Correspondence to: Song Cheol Kim, email: drksc@amc.seoul.kr \\ Keywords: pancreatic ductal adenocarcinoma, pancreatic cancer, DPC4, SMAD4 \\ Received: October 02, $2016 \quad$ Accepted: December 31, 2016 \\ Published: January 30, 2017
}

\section{ABSTRACT}

Objectives: The objective of this study was to investigate the role of genetic status of DPC4 in recurrence patterns of resected pancreatic ductal adenocarcinoma (PDAC).

Methods: Between April 2004 and December 2011, data on patients undergoing surgical resection for PDAC were reviewed. Genetic status of DPC4 was determined and correlated to recurrence patterns and clinical outcomes.

Results: Analysis of 641 patients revealed that genetic status of DPC4 was associated with overall survival and was highly correlated with recurrence patterns, as inactivation of the DPC4 gene was the strongest predictor of metastatic recurrence (odds ratio $\mathbf{~ 4 . 2 8}$ ). Treatment modalities for recurrent PDAC included chemotherapy alone and concurrent chemotherapy along with local control. For both locoregional and metastatic recurrence, local control resulted in improved survival; however, for groups subdivided according to recurrence patterns and genetic status of DPC4, local control contributed to improved survival in locoregional recurrences of patients with expressed DPC4, while chemotherapy alone was sufficient for others.

Conclusions: Genetic status of DPC4 contributes to the recurrence patterns following pancreatectomy, and patients with an initially expressed DPC4 gene receive a greater benefit from intensive local control for locoregional recurrence. The DPC4 gene, therefore, may aid the establishment of treatment strategies for initial adjuvant treatment or for recurrent PDAC.

\section{INTRODUCTION}

Advances in understanding the molecular underpinnings of pancreatic ductal adenocarcinoma (PDAC) have begun to contribute to the development of new approaches to clinical management of this devastating cancer. Although these studies are in their infancy, preliminary findings have supported the efficacy of molecular approaches for treatment of PDAC. The development and growth of PDAC involves various 
genetic alterations in oncogenic activation, loss of tumor suppressor gene function, and the overexpression of receptor-ligand systems. Among the several key genes known to contribute to pancreatic carcinogenesis, genetic alterations in $K$-ras and DPC4/SMAD4 are correlated with patient survival [1-5]. Mutational subtypes of the $K$-ras oncogene have been previously studied for targeted genetic therapy in various cancers, including pancreatic cancer [6-9].

The DPC4 gene, which is inactivated in $55-80 \%$ of PDACs, is one of the major tumor suppressor genes targeted in infiltrating PDAC [4, 10-12]. Loss of DPC4 expression occurs late in neoplastic progression and leads to the development of infiltrating pancreatic cancer at the stage of histologically recognizable carcinoma. DPC4 loss also appears to be associated with tumor progression, patterns of failure, and the epithelial-tomesenchymal transition $[10,13]$. The novel study by Iacobuzio-Donahue et al. demonstrated that the genetic status of DPC4 was correlated with patterns of failure in patients with pancreatic cancer [14]. These investigators performed rapid autopsies on patients with documented pancreatic cancer and found that the histological features and patterns of failure were correlated with the genetic status of DPC4 (i.e., locally destructive tumors in patients with an expressed $D P C 4$ gene vs. distant metastasis in patients with an inactivated DPC4 gene). Based on these findings, Iacobuzio-Donahue and colleagues concluded that determination of the status of DPC4 upon initial diagnosis may aid the stratification of patients into treatment regimens related to local control versus systemic therapy; however, further follow-up prospective studies designed to confirm and extend this finding were proposed.

In terms of treatment, even after curative resection of PDAC, the recurrence rate is very high at early stages, and no effective therapeutic strategies for the treatment of recurrent PDAC have been established to date. To advance the current therapeutic strategies, it is important to determine the factors or treatment modalities that affect prognosis after PDAC recurrence. Therefore, based on the novel findings of Iacobuzio-Donahue et $a l$, we hypothesized that the efficacy of the treatment modality for recurrent PDAC may be closely associated with the biological features of the $D P C 4$ gene. To assess the relationship between the DPC4 gene and both recurrence and treatment, we prospectively collected patient data regarding the initial DPC4 genetic status of PDAC. We reviewed recurrence patterns and responses to treatment modalities according to the genetic status of $D P C 4$. The results of this study indicate that the genetic status of $D P C 4$ plays a key role in the recurrence patterns following pancreatectomy for PDAC and can be used in the establishment of therapeutic strategies for recurrent PDAC.

\section{RESULTS}

\section{Study population}

Clinicopathological features of the study cohort are listed in Table 1 . The 641 patients included 374 men and 267 women with a median age at diagnosis of 61.0 years (range: $22.0-84.0)$. There were 198 (30.9\%) patients with a preoperative history of diabetes mellitus (DM). The preoperative CA19-9 levels were elevated in $405(63.2 \%)$ patients. Patients had disease in the head/ uncinate process of the pancreas $(61.8 \%)$, the body/tail of the pancreas (26.8\%), and the entire pancreas (11.4\%). Pathological reports described 7 (1.1\%) patients with T1 stage disease, 22 (3.4\%) patients with T2, 601 (93.8\%) patients with T3, and $11(1.7 \%)$ patients with T4. Also, $280(43.7 \%)$ patients had N0 stage disease, and 361 (56.3\%) patients had N1 stage disease. Combined major vascular resection was performed in $183(28.5 \%)$ patients. R0 resection was achieved in $548(85.5 \%)$ patients. Most of the tumors were moderately differentiated $(74.3 \%)$, while $12.6 \%$ were poorly differentiated and $10.5 \%$ were well differentiated. Perineural invasion was present in $516(80.5 \%)$ patients, while lymphovascular invasion was present in $266(41.5 \%)$ patients. The DPC4 gene was inactivated in $68.1 \%$ of the study subjects. After pancreatectomy, 374 (58.3\%) and 72 (11.2\%) patients received adjuvant chemotherapy or concurrent chemoradiotherapy, respectively. Table 1 lists the median survival and statistical significance values according to each clinicopathological factor. The overall survival (OS) of patients was significantly associated with the following factors: CA19-9 level, cancer location, T stage, $N$ stage, major vessel resection, resection margin status, tumor differentiation, presence of perineural invasion, presence of lymphovascular invasion, inactivation of the DPC4 gene, and adjuvant therapy.

\section{Analysis of recurrence patterns}

Linear logistic regression analysis was conducted to identify factors that affected recurrence patterns (Table 2). Localization throughout the entire pancreas, inactivation of DPC4 gene function, and no adjuvant therapy were identified as independent factors that determined metastatic recurrence. Among these factors, inactivation of the DPC4 gene was the most strongly correlated with metastatic recurrence (adjusted odds ratio, $[\mathrm{aOR}]=4.28$ ).

\section{DPC4 gene status defines infiltrative or metastatic behavior and affects patient prognoses}

Throughout the study cohort, computed tomography (CT) images of patients obtained between January 2011 and December 2011 were reviewed by a radiologist 
Table 1: Clinicopathological features and survival analysis of all resected pancreatic ductal adenocarcinoma ( $\mathrm{n}=641)$

\begin{tabular}{|c|c|c|c|c|}
\hline Variables & $\mathbf{n}$ & $\%$ & Median survival (months) & $p$ \\
\hline \multicolumn{5}{|l|}{ Clinical factors } \\
\hline \multicolumn{5}{|l|}{ Sex } \\
\hline Male & 374 & 58.3 & 21.9 & \\
\hline Female & 267 & 41.7 & 19.0 & 0.84 \\
\hline \multicolumn{5}{|l|}{ Age, years } \\
\hline Median & \multicolumn{2}{|c|}{61.0} & & 0.15 \\
\hline Range & \multicolumn{2}{|c|}{$22-84$} & & \\
\hline \multicolumn{5}{|l|}{ Preoperative DM } \\
\hline No & 443 & 69.1 & 21.6 & \\
\hline Yes & 198 & 30.9 & 20.0 & 0.61 \\
\hline \multicolumn{5}{|l|}{ CA $19-9^{\dagger}$} \\
\hline Normal $(\leq 37)$ & 226 & 35.3 & 28.2 & \\
\hline Elevated (>37) & 405 & 63.2 & 18.0 & $<0.001$ \\
\hline NA & 10 & 1.5 & & \\
\hline \multicolumn{5}{|l|}{ Tumor factors } \\
\hline \multicolumn{5}{|l|}{ Location of cancer } \\
\hline Head/Uncinate process & 396 & 61.8 & 20.1 & \\
\hline Body/Tail & 172 & 26.8 & 31.7 & 0.04 \\
\hline Entire pancreas & 73 & 11.4 & 11.5 & $<0.001$ \\
\hline \multicolumn{5}{|l|}{ T stage } \\
\hline $\mathrm{T} 1$ & 7 & 1.1 & NA & \\
\hline $\mathrm{T} 2$ & 22 & 3.4 & 34.3 & 0.15 \\
\hline $\mathrm{T} 3$ & 601 & 93.8 & 20.5 & 0.01 \\
\hline $\mathrm{T} 4$ & 11 & 1.7 & 22.3 & 0.16 \\
\hline \multicolumn{5}{|l|}{$\mathrm{N}$ stage } \\
\hline N0 & 280 & 43.7 & 30.0 & \\
\hline N1 & 361 & 56.3 & 17.4 & $<0.001$ \\
\hline \multicolumn{5}{|l|}{ Major vessel resection } \\
\hline No & 458 & 71.5 & 24.3 & \\
\hline Yes & 183 & 28.5 & 15.2 & $<0.001$ \\
\hline \multicolumn{5}{|l|}{ Resection margin status } \\
\hline R0 & 548 & 85.5 & 21.7 & \\
\hline $\mathrm{R} 1$ & 93 & 14.5 & 16.4 & 0.02 \\
\hline \multicolumn{5}{|l|}{ Differentiation $^{\dagger}$} \\
\hline WD & 67 & 10.5 & 33.4 & \\
\hline MD & 476 & 74.3 & 20.5 & 0.03 \\
\hline $\mathrm{PD}$ & 81 & 12.6 & 12.5 & $<0.001$ \\
\hline NA & 17 & 2.6 & & \\
\hline \multicolumn{5}{|l|}{ Perineural invasion } \\
\hline Absent & 125 & 19.5 & 28.2 & \\
\hline Present & 516 & 80.5 & 19.5 & $\begin{array}{l}\mathbf{0 . 0 0 2} \\
\text { (Continued) }\end{array}$ \\
\hline
\end{tabular}




\begin{tabular}{lcccc}
\hline Variables & $\mathbf{n}$ & $\mathbf{\%}$ & Median survival (months) & $\boldsymbol{p}$ \\
\hline Lymphovascular invasion & & & & \\
$\quad$ Absent & 375 & 58.5 & 25.1 & $\mathbf{0 . 0 0 1}$ \\
$\quad$ Present & 266 & 41.5 & 15.7 & \\
DPC4 gene & & & & $\mathbf{0 . 0 3}$ \\
$\quad$ Normal & 165 & 25.7 & 25.4 & \\
$\quad$ Inactivated & 476 & 74.3 & 19.4 & $\mathbf{0 . 0 0 6}$ \\
Adjuvant therapy & & & & $\mathbf{0 . 0 0 4}$ \\
\hline No & 195 & 30.4 & 22.7 & 28.2 \\
CTx alone & 374 & 58.3 & & \\
CCRTx & 72 & 11.2 & & \\
\hline
\end{tabular}

', Some of the data was not available.

DM, diabetes mellitus; WD, well differentiated; MD, moderately differentiated; PD, poorly differentiated; NA, not available; CTx, chemotherapy; CCRTx, concurrent chemo-radiotherapy.

(Hyoung Jung Kim) at our institute. Peripancreatic infiltration was defined as peritumoral fatty stranding, and vascular invasion was evaluated by using the criteria of tumor thrombus, vessel occlusion, stenosis and contour deformity $[15,16]$. CT imaging characteristics were compared according to the genetic status of $D P C 4$ (Table 3). Expressed DPC4 (DPC4+) cancers tended to be welldefined with less peripancreatic infiltration compared to inactivated DPC4 (DPC4-) cancers $(81.3 \%$ vs. $94.3 \%$, $p=0.01$ ); however, major arterial or venous invasions did not differ between the two groups.

Among the 641 resected PDAC patients, 165 $(25.7 \%)$ and $476(74.3 \%)$ patients had expressed and inactivated DPC4 genes, respectively. During the followup period, 500 patients had recurrent disease, including 155 locoregional and 345 metastatic recurrences. Metastatic recurrences of overall patients could be subdivided into localized or diffuse metastases (Figure 1A). The proportion of metastatic recurrence was significantly higher in the DPC4- than in the DPC4+ patients. The most common metastatic site was the liver (31.8\% of all recurrences), and 51 patients had localized hepatic metastasis. The "others" $(16.0 \%)$ included the para-aortic lymph nodes, intestines, and other tissues. According to $D P C 4$ status, there were 68 locoregional and 54 metastatic recurrences in the $D P C 4+$ group, and 87 locoregional and 291 metastatic recurrences in the DPC4group (Figure 1B). Table 4 shows initial sites of recurrence and detailed treatment modalities according to the DPC4 gene status.

The DPC4+ and DPC4- groups were also assessed following restriction of the study population to 500 patients with recurrent PDAC (Table 5). Cancer located throughout the pancreas was $\sim 3$-fold more frequent in the DPC4- group (16.1\%) than in the DPC4+ group (5.7\%), while metastatic recurrence patterns were more dominant in the DPC4- group (77.0\%) than in the $\mathrm{DPC4}$ - group $(44.3 \%)$. Furthermore, the cancer location $(p=0.01)$ and resection margin status $(p=0.05)$ were each associated with $D P C 4$ gene status.

The OS and progression free survival (PFS) of patients were also evaluated based on the genetic status of DPC4 (Figure 2). The median OS was 25.4 and 19.4 months in the $D P C 4+$ and DPC4- groups, respectively $(p=0.02)$, and the median PFS was 11.4 and 8.9 months in the $D P C 4+$ and $D P C 4$ - groups, respectively $(p=0.04)$.

\section{Concurrent local control for recurrent PDAC enhances patient survival only in DPC4+ cancers}

As recurrence patterns are directly associated with treatment strategies, survival after recurrence was compared according to the treatment modality in each recurrence group (Table 6). The application of both chemotherapy (CTx) and local control (LCx) was most effective throughout the entire population: the unadjusted HRs were 0.33 (95\% C.I. $0.20-0.57)$ in the locoregional group and 0.49 (95\% C.I. $0.32-0.75)$ in the metastatic group compared with the untreated group. After adjusting for confounders, CTx was found to be better than no therapy, and the addition of a local control improved survival, irrespective of recurrence patterns. This phenomenon was consistent, even in comparisons between CTx alone and CTx + LCx in both recurrence groups ( $p=0.004$, locoregional; $p=0.04$, metastatic).

To probe the effects of treatment according to DPC4 genetic status, we investigated the correlations between overall survival in each subgroup subdivided by treatment modalities, recurrence patterns, and the DPC4 gene status (Table 7). In all subgroups, the addition of LCx improved the OS. Notably, the unadjusted hazard ratio (uHR) of $\mathrm{CTx}+\mathrm{LCx}$ for locoregional recurrence in 
Table 2: Linear logistic regression identifying factors affecting metastatic recurrence in resected pancreatic ductal adenocarcinoma

\begin{tabular}{|c|c|c|c|c|}
\hline Variables & uOR & $95 \% \mathrm{CI}$ & aOR & $95 \% \mathrm{CI}$ \\
\hline \multicolumn{5}{|l|}{ Clinical factors } \\
\hline \multicolumn{5}{|l|}{ Sex } \\
\hline Male & Reference & & & \\
\hline Female & 0.83 & 0.57 to 1.23 & & \\
\hline \multicolumn{5}{|l|}{ Preoperative DM } \\
\hline No & Reference & & & \\
\hline Yes & 1.14 & 0.76 to 1.73 & & \\
\hline \multicolumn{5}{|l|}{ CA 19-9 } \\
\hline Normal (<37) & Reference & & & \\
\hline Elevated $(>37)$ & 0.88 & 0.58 to 1.33 & & \\
\hline Tumor factors & & & & \\
\hline
\end{tabular}

Location of cancer

Head/Uncinate process

Body/Tail

Reference

Reference

Entire pancreas

1.50

0.95 to 2.37

1.75

1.07 to 2.86

1.82

1.21 to 4.44

2.06

1.04 to 4.07

T stage

$\mathrm{T} 1 / 2$

Reference

T3/4

1.22

0.44 to 3.37

$\mathrm{N}$ stage

N0

N1

Reference

$0.98 \quad 0.67$ to 1.45

Major vessel resection

No

Reference

Yes

$1.11 \quad 0.73$ to 1.68

Resection margin status

R0

Reference

R1

$1.02 \quad 0.59$ to 1.73

Differentiation

WD

Reference

MD

$1.43 \quad 0.76$ to 2.69

PD

2.21

0.98 to 4.98

Perineural invasion

Absent

Reference

Present

1.03

0.63 to 1.68

Lymphovascular invasion

Absent

Reference

Present

$1.06 \quad 0.72$ to 1.55




\begin{tabular}{lcccc}
\hline Variables & uOR & $\mathbf{9 5 \%}$ CI & aOR & $\mathbf{9 5 \%}$ CI \\
\hline $\begin{array}{l}D P C 4 \text { gene } \\
\text { Normal }\end{array}$ & Reference & & Reference & \\
Inactivated & 4.21 & $\mathbf{2 . 7 4}$ to 6.48 & $\mathbf{4 . 2 8}$ & $\mathbf{2 . 7 5}$ to $\mathbf{6 . 6 8}$ \\
Adjuvant therapy & & & & \\
\hline No & Reference & & Reference & \\
CTx alone & $\mathbf{0 . 5 8}$ & $\mathbf{0 . 3 7}$ to $\mathbf{0 . 9 2}$ & $\mathbf{0 . 5 1}$ & $\mathbf{0 . 3 2}$ to $\mathbf{0 . 8 4}$ \\
CCRTx & 0.73 & 0.36 to 1.48 & 0.82 & 0.38 to 1.74 \\
\hline
\end{tabular}

uOR, unadjusted odds ratio; aOR, adjusted odds ratio; DM, diabetes mellitus; WD, well differentiated; MD, moderately differentiated; PD, poorly differentiated; CTx, chemotherapy; CCRTx, concurrent chemo-radiotherapy.

Table 3: Correlation of CT imaging characteristics with DPC4 gene status in the patients diagnosed as pancreatic ductal adenocarcinoma between January 2011 and December 2011

\begin{tabular}{lcccc}
\hline Variables & & $\begin{array}{c}\text { DPC4+ } \\
(\mathbf{n = 7 5 )}\end{array}$ & $\begin{array}{c}\text { DPC4- } \\
(\mathbf{n = 8 8})\end{array}$ & $\boldsymbol{p}$ \\
\hline Peripancreatic infiltration & Present & $61(81.3 \%)$ & $83(94.3 \%)$ & $\mathbf{0 . 0 1}$ \\
& Absent & $14(18.7 \%)$ & $5(5.7 \%)$ & \\
Artery invasion & Present & $23(30.7 \%)$ & $35(39.8 \%)$ & 0.23 \\
& Absent & $52(69.3 \%)$ & $53(60.2 \%)$ & \\
Vein invasion & Present & $23(30.7 \%)$ & $32(36.4 \%)$ & 0.44 \\
& Absent & $52(69.3 \%)$ & $56(63.6 \%)$ & \\
\hline
\end{tabular}

a

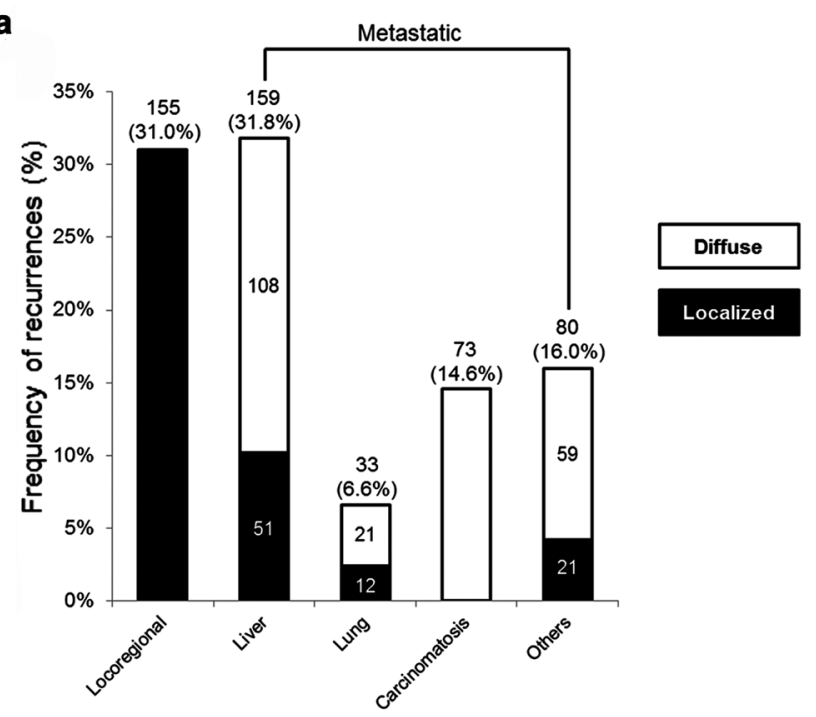

b

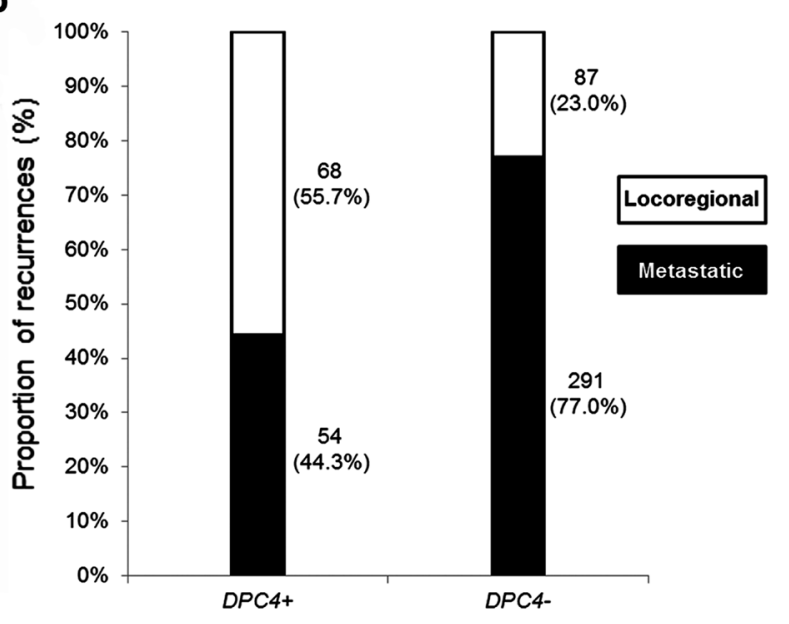

Figure 1: Diagrams for recurrence patterns of overall patients and proportion of recurrences according to the $D P C 4$ status. a. Frequency of recurrence following pancreatectomy for pancreatic ductal adenocarcinoma (PDAC). The liver was the most common site of metastasis. b. Proportion of recurrences stratified by DPC4 gene status. The study cohort consisted of 500 patients with recurrent disease in 68 locoregional and 54 metastatic recurrences in the DPC4+ group and in 87 locoregional and 291 metastatic recurrences in the DPC4- group. 
Table 4: Initial sites of recurrence and detailed treatment modalities according to the $D P C 4$ gene status $(\mathrm{n}=500)$

\begin{tabular}{|c|c|c|c|c|c|c|c|c|c|}
\hline \multirow{2}{*}{ c } & \multirow[b]{3}{*}{$\mathbf{n}$} & \multirow[b]{3}{*}{$\%$} & \multicolumn{7}{|c|}{ Treatment for recurrent disease } \\
\hline & & & \multicolumn{5}{|c|}{ Chemotherapy + Local control } & \multirow[b]{2}{*}{ Chemotherapy alone } & \multirow{2}{*}{$\begin{array}{c}\text { No } \\
\text { therapy }\end{array}$} \\
\hline $\begin{array}{l}\text { Initial site of } \\
\text { recurrence }\end{array}$ & & & Operation & RFA & TACI & TACE & $\mathbf{R T x}$ & & \\
\hline \multicolumn{10}{|l|}{$\mathrm{DPC} 4+$} \\
\hline Locoregional & 68 & 13.6 & 5 & - & - & - & 7 & 39 & 17 \\
\hline \multicolumn{10}{|l|}{ Metastatic } \\
\hline Liver & 30 & 6.0 & 2 & 5 & - & - & 2 & 13 & 8 \\
\hline Lung & 7 & 1.4 & 1 & - & - & - & - & 4 & 2 \\
\hline $\begin{array}{l}\text { Peritoneal } \\
\text { carcinomatosis }\end{array}$ & 9 & 1.8 & - & - & - & - & - & 6 & 3 \\
\hline Others & 8 & 1.6 & - & - & - & - & 3 & 2 & 3 \\
\hline \multicolumn{10}{|l|}{$D P C 4-$} \\
\hline Locoregional & 87 & 17.4 & 4 & - & - & - & 12 & 46 & 25 \\
\hline \multicolumn{10}{|l|}{ Metastatic } \\
\hline Liver & 129 & 25.8 & 4 & 16 & 1 & 1 & 2 & 70 & 35 \\
\hline Lung & 26 & 5.2 & 5 & - & - & - & 2 & 14 & 5 \\
\hline $\begin{array}{l}\text { Peritoneal } \\
\text { carcinomatosis }\end{array}$ & 64 & 12.8 & - & - & - & - & - & 36 & 28 \\
\hline Others & 72 & 14.4 & 2 & - & - & - & 8 & 34 & 28 \\
\hline
\end{tabular}

RFA, radiofrequency ablation; TACI, transarterial chemoinfusion; TACE, transarterial chemoembolization; RTx, radiotherapy.

the DPC4+ subgroup was 0.25 (95\% C.I. 0.10-0.61) and the aHR was 0.24 (95\% C.I. 0.10-0.59) after adjusting for confounders. Comparisons between CTx alone and CTx + LCx revealed that the addition of local control for locoregional recurrence in the $D P C 4+$ group, but not in the DPC4- group, yielded the greatest benefit $(p=0.002)$ in improving survival. Figure 3 also shows survival benefit of local control for locoregional recurrence in $\mathrm{DPC} 4+$ group. Therefore, the effects of treatment modalities could be influenced by recurrence patterns according to the genetic status of DPC4.

\section{DISCUSSION}

Without surgical resection, PDAC is often incurable. Even after surgery, early recurrence or metastasis frequently occur, and the overall survival rate remains low. These characteristics usually discourage efforts to treat this disease. As shown by the current findings, most of the clinicopathological features of patients with PDAC result in a dismal prognosis. Although adjuvant therapies, including CTx and/or radiotherapy, have shown the potential to prevent or cure disease, many limitations and failures of these therapies are evident in the observation that $500(78.0 \%)$ of 641 patients exhibited recurrent cancer during our study period. Accordingly, we should focus our attention on treatments for both recurrent and primary PDAC.

In this current era of molecular biology, a better understanding of the cellular and molecular features of cancer may yield major advances in its clinical management. In the present study, we investigated correlations between the genetic status of DPC4 and the postoperative clinical course in a large cohort of PDAC cases. Furthermore, we demonstrated the importance of the DPC4 gene in tumor progression following surgical resection and investigated treatment status for recurrent cancer in correlation with the DPC4 status.

In agreement with these results, several reports [2-4, $17,18]$ have shown that DPC4 gene status was associated with patient prognoses; however, no direct correlation at the molecular level has yet been established. SMAD4 (DPC4) plays an important role in both tumor suppression and progression [19]. Sustained exposure to the cytokine transforming growth factor- $\beta$ (TGF- $\beta$ ), which leads to the epithelial-to-mesenchymal transition (EMT) and the 
Table 5: Correlation between clinicopathological features and the status of DPC4 gene in patients with recurrent cancer $(\mathbf{n}=\mathbf{5 0 0})$

\begin{tabular}{|c|c|c|c|}
\hline Variables & $D P C 4+(\%)$ & DPC4- (\%) & $p$ \\
\hline Recurrence patterns & & & $<0.001$ \\
\hline Locoregional & $68(55.7)$ & $87(23.0)$ & \\
\hline Metastatic & $54(44.3)$ & $291(77.0)$ & \\
\hline \multicolumn{4}{|l|}{ Clinical factors } \\
\hline$\overline{\operatorname{Sex}}$ & & & 0.99 \\
\hline Male & $73(59.8)$ & $226(59.8)$ & \\
\hline Female & $49(40.2)$ & $152(40.2)$ & \\
\hline Age, years & & & 0.72 \\
\hline Mean \pm SD & $59.6 \pm 9.3$ & $60.0 \pm 10.2$ & \\
\hline Preoperative DM & & & 0.53 \\
\hline Absent & $35(28.7)$ & $120(31.7)$ & \\
\hline Present & $87(71.3)$ & $258(68.3)$ & \\
\hline CA19-9 $(n=491)$ & & & 0.87 \\
\hline Normal $(\leq 37 \mathrm{U} / \mathrm{mL})$ & $39(32.0)$ & $115(31.2)$ & \\
\hline Elevated (> $37 \mathrm{U} / \mathrm{mL}$ ) & $83(68.0)$ & $254(68.8)$ & \\
\hline \multicolumn{4}{|l|}{ Tumor factors } \\
\hline Location of cancer & & & 0.01 \\
\hline Head/Uncinate process & $79(64.8)$ & $226(59.8)$ & \\
\hline Body/Tail & $36(29.5)$ & $91(24.1)$ & \\
\hline Entire pancreas & $7(5.7)$ & $61(16.1)$ & \\
\hline T stage & & & $0.597 \dagger$ \\
\hline $\mathrm{T} 1 / 2$ & $4(3.3)$ & $13(3.4)$ & \\
\hline $\mathrm{T} 3 / 4$ & $118(96.7)$ & $365(96.6)$ & \\
\hline $\mathrm{N}$ stage & & & 0.93 \\
\hline No & $48(39.3)$ & $147(38.9)$ & \\
\hline $\mathrm{N} 1$ & $74(60.7)$ & $231(61.1)$ & \\
\hline Major vessel resection & & & 0.264 \\
\hline No & $89(73.0)$ & $255(67.5)$ & \\
\hline Yes & $33(27.0)$ & $123(32.5)$ & \\
\hline Resection margin status & & & 0.05 \\
\hline $\mathrm{R} 0$ resection & $97(79.5)$ & $328(86.8)$ & \\
\hline $\mathrm{R} 1$ resection & $25(20.5)$ & $50(13.2)$ & \\
\hline Differentiation $(n=488)$ & & & 0.17 \\
\hline WD & $16(13.7)$ & $30(8.1)$ & \\
\hline MD & $83(70.9)$ & $288(77.6)$ & \\
\hline $\mathrm{PD}$ & $18(15.4)$ & $53(14.3)$ & \\
\hline Perineural invasion & & & 0.15 \\
\hline Absent & $27(22.1)$ & $62(16.4)$ & \\
\hline Present & $95(77.9)$ & $316(83.6)$ & \\
\hline Lymphovascular invasion & & & 0.48 \\
\hline Absent & $64(52.5)$ & $212(56.1)$ & \\
\hline Present & $58(47.5)$ & $166(43.9)$ & \\
\hline \multicolumn{4}{|l|}{ Adjuvant therapy } \\
\hline No & $31(25.4)$ & $109(28.8)$ & 0.12 \\
\hline CTx alone & $72(59.0)$ & $234(61.9)$ & \\
\hline CCRTx & $19(15.6)$ & $35(9.3)$ & \\
\hline
\end{tabular}

†, Fisher's exact test;

$\mathrm{SD}$, standard deviation; $\mathrm{DM}$, diabetes mellitus; $\mathrm{WD}$, well differentiated; $\mathrm{MD}$, moderately differentiated; PD, poorly differentiated; CTx, chemotherapy; CCRTx, concurrent chemo-radiotherapy. 
inhibition of growth arrest and apoptosis, suppresses Smad signaling [20]. Additionally, the Smad proteins play a central role in TGF- $\beta$-dependent EMT associated with tumor progression and metastasis [21]. Yamada et al reported that patients with epithelial tumors had a better OS than mesenchymal-type tumors, which often lack DPC4 expression, and showed that EMT was the most significant independent prognostic factor for pancreatic cancer $[13,22]$.

In a clinical setting, Iacobuzio-Donahue et al [14] reported that the initial DPC4 genetic status in PDAC was correlated with patterns of failure, which were locally destructive or metastatic tumors; however, these investigators concluded that further follow-up prospective studies were needed. Our present study also revealed that the genetic status of DPC4 highly reflected clinical features and initial recurrence patterns following pancreatectomy: an expressed DPC4 gene was associated with locoregional recurrence, and inactivation of DPC4 was correlated with metastatic recurrence. At our institute, we performed repeated resections or locally targeted treatments (radiofrequency ablation, transarterial chemoembolization or radiotherapy) with the consent of patients if the lesion was confined to a locoregional area or was metastatic. The feasibility of repeated resection for recurrent PDAC after initial pancreatectomy is not yet accepted; however, several previous studies [23-25] support the concept of repeated local therapy for either locoregional or metastatic recurrences. Analysis of the effects of intensive local therapy, including repeated resection, ablation, and radiotherapy, for recurrent $\mathrm{PDAC}$ indicated that the application of both CTx and LCx was most effective followed by CTx alone. The gradient of survival risk was precipitous in the locoregional recurrence group compared with the metastatic group. Although systemic CTx is a well-established treatment of choice for recurrent PDAC, we found that the addition of intensive local therapy contributes to improved survival. Therefore, diminishing the tumor burden may create a synergistic effect to improve survival in cooperation with systemic CTx. Comparison of OS according to the genetic status of DPC4 and treatment modalities led to a better understanding of the role of local therapy (Table 7). In this analysis, concurrent local control was found to be effective for locoregional recurrence in $\mathrm{DPC} 4+$ cancers but not for metastatic recurrence or in DPC4- cancers. To calibrate biases caused by differences in the severity of the metastatic burden, we conducted subgroup analysis for potentially resectable (localized) metastasis (Figure 4). The median survival following CTx $+\mathrm{LCx}$ and CTx alone for potentially resectable metastases was 23.6 and 30.1 months in DPC4+ cancers $(p=0.82)$, respectively and 22.8 and 20.7 months in DPC4- cancers $(p=0.25)$, respectively. These findings confirmed our hypothesis that the effect of local control may be maximized in locoregional recurrence with an expressed DPC4 gene. Based on the correlation of the genetic status of DPC4 with recurrence patterns and the role of local control, we suggest the use of a treatment algorithm for recurrent PDAC during surveillance following pancreatectomy (Figure 5).

A previous study reported that DPC4 failed to predict recurrence pattern. Winter et al reported in their a

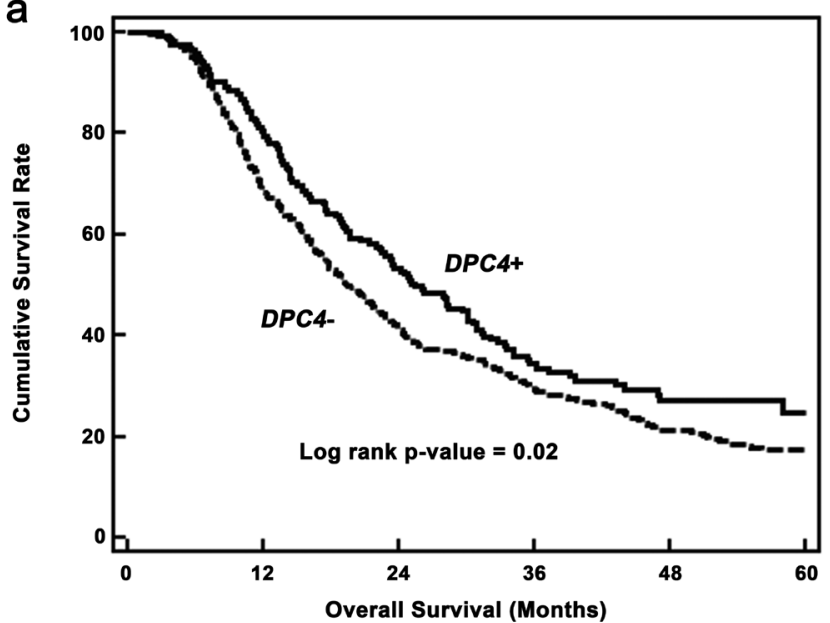

No. at risk

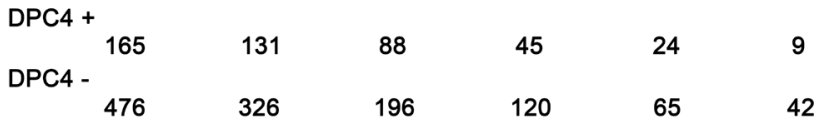

b

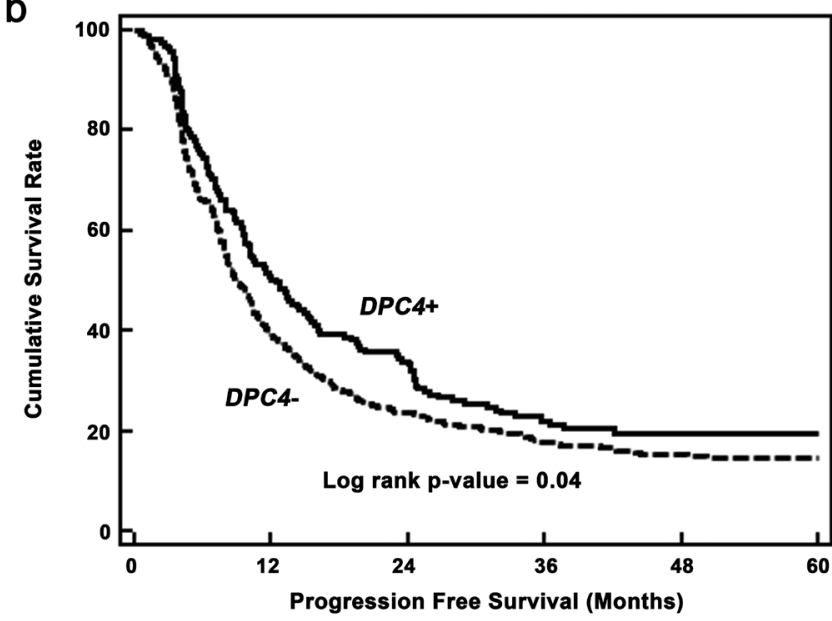

No. at risk

$\begin{array}{lccccc}\text { DPC4 + } & & & & & \\ 165 & 83 & 55 & 27 & 15 & 5 \\ \text { DPC4 - } & & 112 & 70 & 41 & 27\end{array}$

Figure 2: Kaplan-Meier survival curves. a. Overall survival based on the genetic status of $D P C 4$ (n=641). Median overall survival of patients with $D P C+$ and $D P C$ - cancers were 25.4 and 19.4 months ( $p=0.02)$, respectively. b. Progression-free survival based on the genetic status of $D P C 4$. The median progression free survival of $D P C 4+$ and $D P C 4$ - cancers were 11.4 and 8.9 months $(p=0.04)$, respectively. 
Table 6: Analysis for survival after recurrence according to the recurrence patterns and treatment modalities $(\mathbf{n}=\mathbf{5 0 0})$

\begin{tabular}{|c|c|c|c|c|c|c|c|}
\hline Variables & uHR & $95 \% \mathrm{CI}$ & $\begin{array}{l}p \text { for } \\
\text { trend }\end{array}$ & $\mathbf{a H R}^{\dagger}$ & $95 \%$ CI & $\begin{array}{l}p \text { for } \\
\text { trend }\end{array}$ & $\begin{array}{c}p \text { for CTx alone } \\
\text { vs. CTx }+\mathrm{LCx}\end{array}$ \\
\hline \multicolumn{8}{|c|}{ Locoregional recurrence } \\
\hline No therapy & Reference & & $<0.001$ & Reference & & $<\mathbf{0 . 0 0 1}$ & \\
\hline CTx alone & 0.61 & 0.42 to 0.90 & & 0.60 & 0.41 to 0.88 & & 0.004 \\
\hline $\mathrm{CTx}+\mathrm{LCx}$ & 0.33 & 0.20 to 0.57 & & 0.35 & 0.20 to 0.59 & & \\
\hline \multicolumn{8}{|c|}{ Metastatic recurrence } \\
\hline No therapy & 1.23 & 0.86 to 1.77 & $<0.001$ & 1.19 & 0.82 to 1.71 & $<0.001$ & \\
\hline CTx alone & 0.72 & 0.51 to 1.01 & & 0.68 & 0.48 to 0.96 & & 0.04 \\
\hline $\mathrm{CTx}+\mathrm{LCx}$ & 0.49 & 0.32 to 0.75 & & 0.51 & 0.34 to 0.78 & & \\
\hline
\end{tabular}

$\dagger$, Adjusted with the confounders (Location of cancer and resection margin status)

uHR, unadjusted hazard ratio; aHR, adjusted hazard ratio; CTx, chemotherapy; CTx+LCx, chemotherapy and local control

Table 7: Analysis for overall survival according to the recurrence patterns and treatment modalities in relations with the $D P C 4$ gene status $(\mathrm{n}=500)$

\begin{tabular}{|c|c|c|c|c|c|c|c|}
\hline Variables & uHR & $95 \% \mathrm{CI}$ & $\begin{array}{l}p \text { for } \\
\text { trend }\end{array}$ & $\mathbf{a H R}^{\dagger}$ & $95 \% \mathrm{CI}$ & $\begin{array}{l}p \text { for } \\
\text { trend }\end{array}$ & $\begin{array}{c}p \text { for } \mathrm{CTx} \text { alone } \\
\text { vs. CTx }+\mathrm{LCx}\end{array}$ \\
\hline \multicolumn{8}{|l|}{$D P C 4+$} \\
\hline \multicolumn{8}{|c|}{ Locoregional recurrence } \\
\hline No therapy & Reference & & $<0.001$ & Reference & & $<0.001$ & \\
\hline CTx alone & 0.73 & 0.41 to 1.31 & & 0.68 & 0.37 to 1.22 & & 0.002 \\
\hline $\mathrm{CTx}+\mathrm{LCx}$ & 0.25 & 0.10 to 0.61 & & 0.24 & 0.10 to 0.59 & & \\
\hline \multicolumn{8}{|c|}{ Metastatic recurrence } \\
\hline No therapy & 2.04 & 1.01 to 4.13 & $<0.001$ & 1.99 & 0.97 to 4.06 & $<0.001$ & \\
\hline CTx alone & 0.75 & 0.40 to 1.42 & & 0.80 & 0.42 to 1.12 & & 0.12 \\
\hline $\mathrm{CTx}+\mathrm{LCx}$ & 0.39 & 0.17 to 0.88 & & 0.38 & 0.17 to 0.89 & & \\
\hline \multicolumn{8}{|l|}{$D P C 4-$} \\
\hline \multicolumn{8}{|c|}{ Locoregional recurrence } \\
\hline No therapy & 0.94 & 0.50 to 1.76 & 0.01 & 0.85 & 0.45 to 1.60 & $<0.001$ & \\
\hline CTx alone & 0.59 & 0.33 to 1.05 & & 0.53 & 0.30 to 0.95 & & 0.32 \\
\hline $\mathrm{CTx}+\mathrm{LCx}$ & 0.44 & 0.21 to 0.89 & & 0.39 & 0.19 to 0.81 & & \\
\hline \multicolumn{8}{|c|}{ Metastatic recurrence } \\
\hline No therapy & 1.28 & 0.75 to 2.17 & 0.01 & 1.17 & 0.69 to 2.00 & $<0.001$ & \\
\hline CTx alone & 0.83 & 0.49 to 1.39 & & 0.68 & 0.40 to 1.15 & & 0.12 \\
\hline $\mathrm{CTx}+\mathrm{LCx}$ & 0.55 & 0.31 to 0.99 & & 0.53 & 0.29 to 0.96 & & \\
\hline
\end{tabular}

${ }^{\dagger}$, Adjusted with the confounders (Location of cancer and resection margin status)

uHR, unadjusted hazard ratio; aHR, adjusted hazard ratio; CTx, chemotherapy; CTx $+\mathrm{LCx}$, chemotherapy and local control 
analysis with 127 resected PDAC that loss of DPC4 expression was $31.5 \%$, and it was neither associated with recurrence pattern nor associated with early death [26]. However, the loss rate was quite different from other previous reports showing that DPC4 was lost in 55 up to $80 \%$ of PDAC $[2,4,5,10,13,27,28]$. Although the findings of the present study showed importance of DPC4 status in recurrence, we acknowledge that the recurrence patterns are not solely affected by DPC4 status, and there has been still controversy in the prognostic value of $D P C 4$ status.

This study has selection bias, which is one possible limitation of our study, may have been present in the treatment plans for recurrent PDAC due to differences in prescribed treatment strategies among physicians of various subspecialties, such as surgeons, gastroenterologists, and oncologists. And there were limitations that exact role of each local procedure could not be identified because the number of each LCx was a

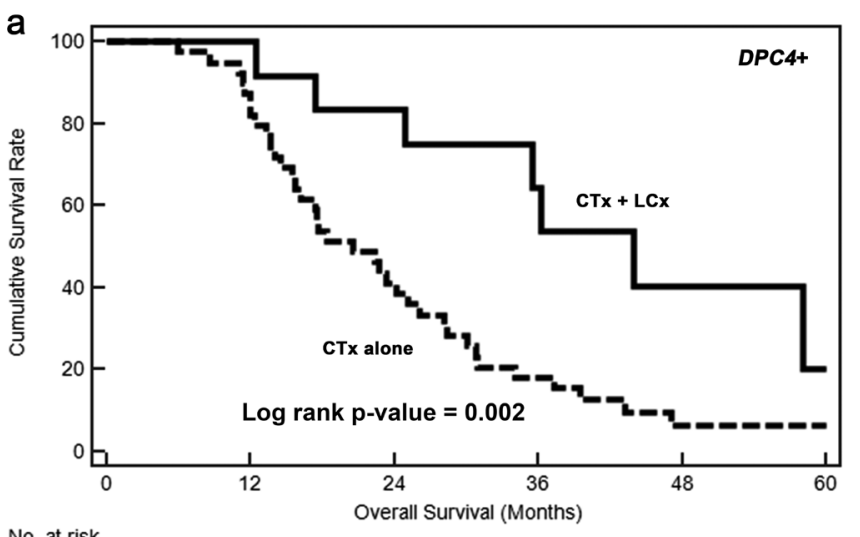

No. at risk

CTx alone

39
CTX + LCx
12
16

10
6

1 b

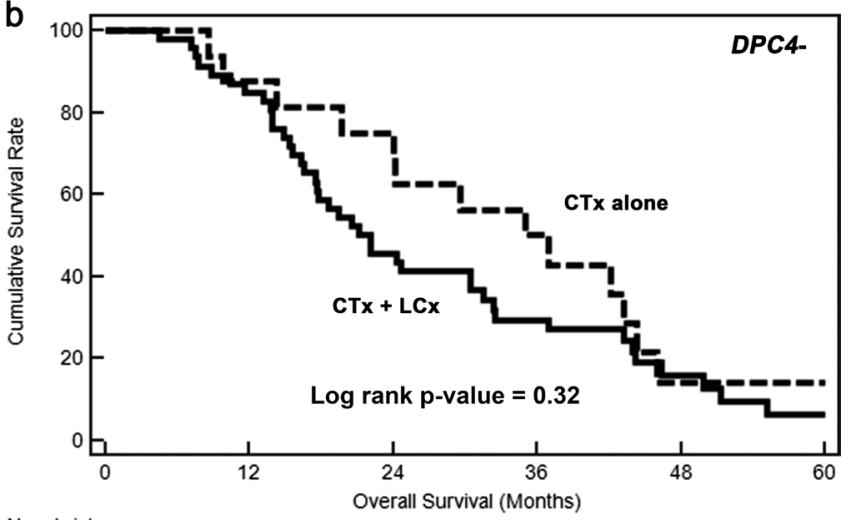

No. at risk CTx alone

CTx + LCx

39

14

Figure 3: Kaplan-Meier survival curves for locoregional recurrences. a. The median overall survival of concurrent chemotherapy and local control (CTx + LCx) versus chemotherapy alone (CTx alone) in DPC4+ cancers were 44.0 versus 20.5 months $(p$ $=0.002)$, respectively. $\mathbf{b}$. The median overall survival of CTx + LCx versus CTx only were 21.1 versus 35.1 months $(p=0.32)$, respectively.

a

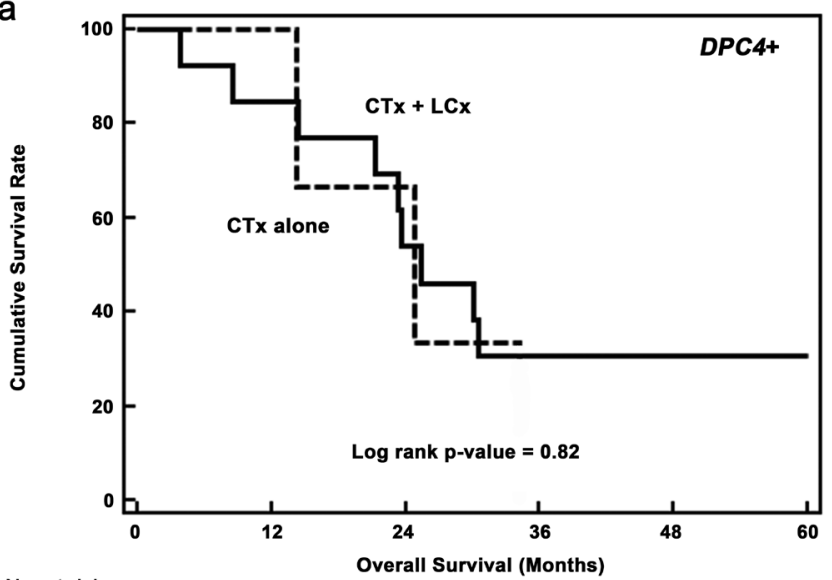

No. at risk

CTx alone

CTX + LCx

$\begin{array}{llll}2 & 0 & 0 & 0 \\ 7 & 4 & 3 & 1\end{array}$

Overall Survival (Months) b

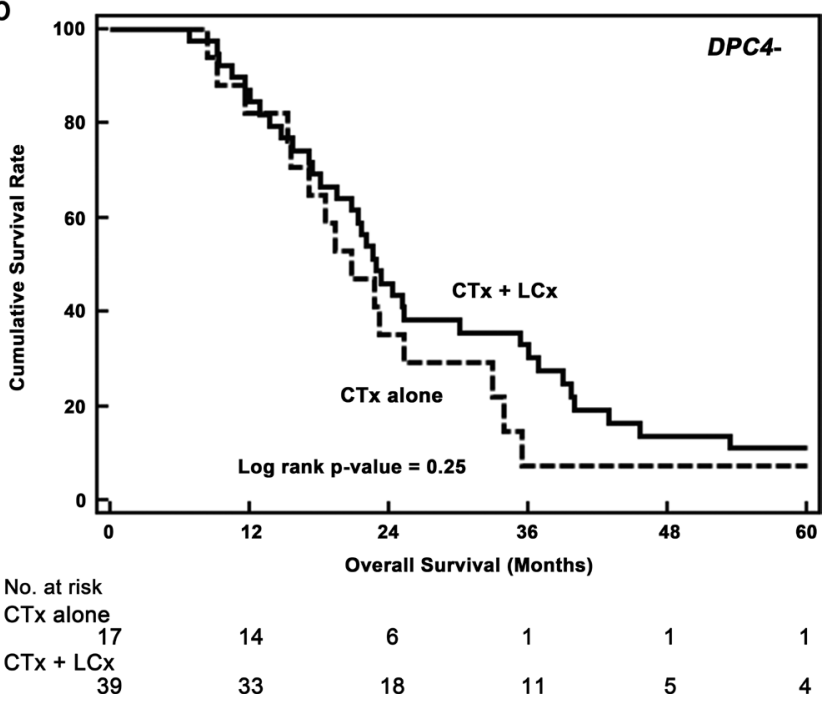

Figure 4: Kaplan-Meier survival curves for potentially resectable (localized) metastatic recurrences. a. The median overall survival of concurrent chemotherapy and local control (CTx $+\mathrm{LCx}$ ) versus chemotherapy alone (CTx alone) in DPC4+ cancers were 23.6 versus 30.1 months $(p=0.82)$, respectively. $\mathbf{b}$. The median overall survival of CTx + LCx versus CTx only were 22.8 versus 20.7 months $(p=0.25)$, respectively. 


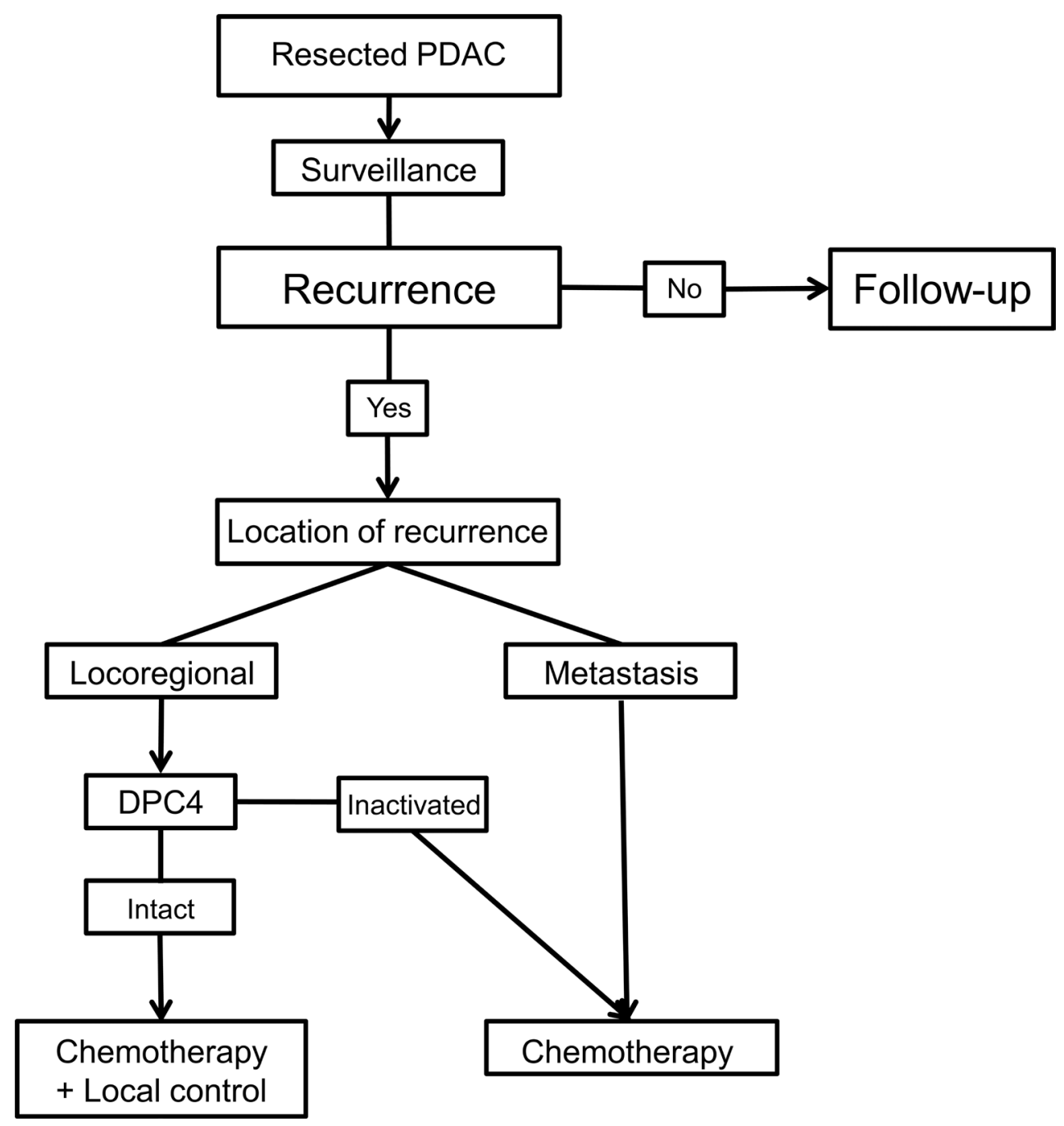

Figure 5: Suggested treatment algorithm for the recurrence of resected pancreatic ductal adenocarcinoma (PDAC) during surveillance.
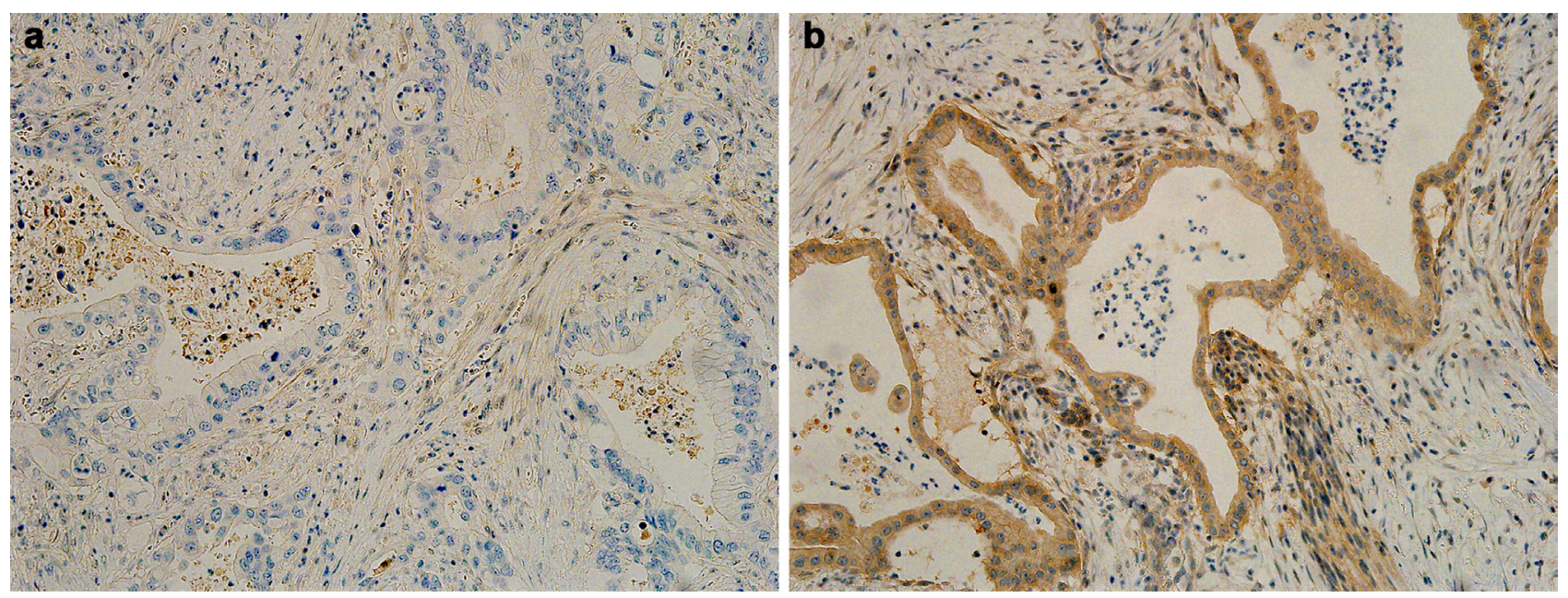

Figure 6: Representative photographs of the immunohistochemistry analysis of DPC4 in pancreatic ductal adenocarcinoma. a. Negative staining indicates an inactivated DPC4 gene. b. Positive staining indicates an expressed DPC4 gene. All photographs are shown at a $\times 200$ magnification. 
small. In addition, the genetic data have been collected prospectively, but this is still a retrospective review of the cases. Therefore, additional studies will be required to verify our present findings. Nonetheless, as our current analysis was conducted with the largest cohort reported to date at a high-volume center with well-established treatment guidelines, our findings provide significant insight into DPC4 gene function in terms of recurrence patterns and treatment plans for recurrent PDAC.

In conclusion, our study of more than 500 patients with PDAC examined the correlations between the clinical course of PDAC and the initial genetic status of DPC4. Our findings suggest clinical relevance of the genetic status of DPC4 in terms of distinct features, including infiltrative features and recurrence patterns, as well as responses to treatment modalities. The genetic status of $D P C 4$ contributes to the recurrence patterns observed follow pancreatectomy for PDAC, and patients with an initially expressed $D P C 4$ gene receive greater benefits from intensive local control therapy for locoregional recurrence. Therefore, studies of the genetic status of $D P C 4$ will help to establish treatment strategies for either the adjuvant setting or recurrent PDAC.

\section{MATERIALS AND METHODS}

\section{Patients}

Between April 2004 and December 2011, a total of 689 consecutive patients with PDAC underwent surgical resection at Asan Medical Center (Seoul, South Korea). Patient data, including genetic alterations, were prospectively collected and retrospectively reviewed using electronic medical records available at our institute. This study was approved by our Institutional Review Board, and all genetic studies were performed after obtaining informed consent. Among the 689 PDAC cases, 26 had stage IV disease, 7 died of other causes, and 15 were lost to follow-up. As a result, 641 patients were included in the current analyses. The margin status of resected specimen was reviewed, and R1 was defined as a distance of the tumor from the resection margin of $\leq 1 \mathrm{~mm}[29,30]$.

\section{Detection of genetic alterations of the DPC4 gene}

As we described previously [4], the genetic status of DPC4 was assessed by immunohistochemical staining. After deparaffinization and antigenic retrieval, slides were labeled with a monoclonal antibody to DPC4 (clone EP618Y, diluted 1:100; Abcam Inc., Cambridge, MA, USA). Labeling was achieved using the avidinbiotin complex method. The chromogen 3-amino-9ethylcarbazole was used. Normal saline was used as a substitute for the primary antibody as a negative control. A single pathologist interpreted and scored the immunohistochemistry staining of DPC4. In pathology reports of slides stained using immunohistochemistry, the frequency of DPC4-positive cells in a tumor population were scored as 0 to 3 as follows: 0 , less than $10 \% ; 1,10 \%$ to $33 \%$ positive; $2,34 \%$ to $67 \%$ positive; and 3 , more than $67 \%$ positive. After scoring, cases were dichotomized as intact/decreased DPC4 expression (score 1-3) and total loss of DPC4 expression (score 0). Representative photographs of immunohistochemistry staining are shown in Figure 6. Negative staining indicated an inactivated DPC4 gene (Figure 6A), and positive staining indicated an expressed DPC4 gene (Figure 6B).

\section{Adjuvant therapy, postoperative surveillance, and detection of the primary recurrent site}

Postoperative adjuvant treatment was administered between 3 weeks and 3 months after surgery. Patients received either 5-fluorouracil with leucovorin or gemcitabine for 6 months. In patients with microscopic residual disease (R1), 5-fluorouracil-based chemoradiation was added. Contrast-enhanced abdominoperineal CT was used for postoperative surveillance, and CA 19-9 levels were examined every 3 months for the first 2 years following surgery and then every 6 months. Diagnoses of locoregional recurrence, which included the region of the pancreatic bed, the root of the mesentery, and hepatoduodenal ligament, were based on progressive soft tissue growth at specific sites and elevated CA19-9 levels [31]. Metastatic recurrence was defined as recurrence in the peritoneal cavity or other remote organs, including the liver, lung, or other organs. When lesions of potential recurrent disease were detected, ${ }^{18} \mathrm{~F}$-fluorodeoxyglucose positron emission tomography (FDG-PET), chest CT, and/or biopsy were performed to confirm the diagnosis of recurrence. Cases with simultaneous locoregional and metastatic recurrences were identified as metastatic recurrence. Metastatic recurrence was sometimes subdivided into localized or diffuse metastases. Localized metastasis indicated metastasized lesion(s) in a focal area, such like a single lobe of liver or lung. Diffuse metastasis indicated lesions throughout multiple areas.

\section{Treatment modalities for recurrent disease}

According to the therapeutic guidelines of our institute, patients with recurrent PDAC are candidates for systemic chemotherapy if the performance status allows. Additionally, aggressive LCx is also employed if the recurrent PDAC is locally controllable. LCx includes complete total pancreatectomy, tumorectomy, hepatic resection, pulmonary resection, radiofrequency ablation, transarterial chemoembolization, and radiotherapy. In the present study, the treatment modalities for recurrence were subdivided into the following three groups: conservative management (no treatment), CTx alone, and concurrent CTx and LCx. 


\section{Statistical analysis}

Univariable comparisons of estimated survival according to clinicopathological factors and genetic alterations were performed using the Kaplan-Meier method and log-rank test. A linear logistic regression model was used to identify factors that affected recurrence patterns, and a descriptive analysis was conducted to examine the relationships between the DPC4 genetic status and either recurrence or survival. Multiple Cox proportional hazard models were used to assess associations between the status of the DPC4 gene and either recurrence patterns or kinetics according to survival after adjusting for covariates, including clinically important confounders that were selected using statistical analyses. The $p$-value for each trend was calculated by treating the treatment modality group as an ordinal variable (i.e., with three different levels: 0 as no treatment, 1 as CTx alone, and 2 as CTx and LCx) with or without DPC4 status (i.e., 0 as expressed, and 1 as deletion). All analyses were performed using SAS (v9.3, SAS Institute, Cary, NC), and a $p$-value $<0.05$ indicated a statistically significant difference.

\section{ACKNOWLEDGMENTS}

This study was supported by a grant of the Korean Health Technology R\&D Project, Ministry of Health \& Welfare, Republic of Korea (grant number: HI14C2640), and this study was also supported by a grant (16-985) from the Asan Institute for Life Sciences, Asan Medical Center, Seoul, Korea. The funders had no role in study design, data collection and analysis, decision to publish, or preparation of the manuscript.

\section{CONFLICTS OF INTEREST}

The authors declare no conflicts of interest.

\section{FUNDING}

The authors have no other funding to disclose.

\section{REFERENCES}

1. Kawesha A, Ghaneh P, Andren-Sandberg A, Ograed D, Skar R, Dawiskiba S, Evans JD, Campbell F, Lemoine $\mathrm{N}$, Neoptolemos JP. K-ras oncogene subtype mutations are associated with survival but not expression of $\mathrm{p} 53$, p16(INK4A), p21(WAF-1), cyclin D1, erbB-2 and erbB-3 in resected pancreatic ductal adenocarcinoma. Int J Cancer. 2000; 89:469-474.

2. Tascilar M, Skinner HG, Rosty C, Sohn T, Wilentz RE, Offerhaus GJ, Adsay V, Abrams RA, Cameron JL, Kern SE, Yeo CJ, Hruban RH, Goggins M. The SMAD4 protein and prognosis of pancreatic ductal adenocarcinoma. Clin Cancer Res. 2001; 7:4115-4121.
3. Biankin AV, Morey AL, Lee CS, Kench JG, Biankin SA, Hook HC, Head DR, Hugh TB, Sutherland RL, Henshall SM. DPC4/Smad4 expression and outcome in pancreatic ductal adenocarcinoma. J Clin Oncol. 2002; 20:4531-4542.

4. Shin SH, Kim SC, Hong SM, Kim YH, Song KB, Park KM, Lee YJ. Genetic alterations of K-ras, p53, c-erbB-2, and DPC4 in pancreatic ductal adenocarcinoma and their correlation with patient survival. Pancreas. 2013; 42:216-222.

5. Oshima M, Okano K, Muraki S, Haba R, Maeba T, Suzuki $\mathrm{Y}$, Yachida S. Immunohistochemically detected expression of 3 major genes (CDKN2A/p16, TP53, and SMAD4/ DPC4) strongly predicts survival in patients with resectable pancreatic cancer. Ann Surg. 2013; 258:336-346.

6. Karapetis CS, Khambata-Ford S, Jonker DJ, O'Callaghan CJ, Tu D, Tebbutt NC, Simes RJ, Chalchal H, Shapiro JD, Robitaille S, Price TJ, Shepherd L, Au HJ, et al. K-ras mutations and benefit from cetuximab in advanced colorectal cancer. N Engl J Med. 2008; 359:1757-1765.

7. Diep CH, Munoz RM, Choudhary A, Von Hoff DD, Han H. Synergistic effect between erlotinib and MEK inhibitors in KRAS wild-type human pancreatic cancer cells. Clin Cancer Res. 2011; 17:2744-2756.

8. Lim SM, Westover KD, Ficarro SB, Harrison RA, Choi HG, Pacold ME, Carrasco M, Hunter J, Kim ND, Xie T, Sim T, Janne PA, Meyerson M, et al. Therapeutic targeting of oncogenic K-Ras by a covalent catalytic site inhibitor. Angew Chem Int Ed Engl. 2014; 53:199-204.

9. Rudolph J, Stokoe D. Selective inhibition of mutant Ras protein through covalent binding. Angew Chem Int Ed Engl. 2014; 53:3777-3779.

10. Wilentz RE, Iacobuzio-Donahue CA, Argani P, McCarthy DM, Parsons JL, Yeo CJ, Kern SE, Hruban RH. Loss of expression of Dpc4 in pancreatic intraepithelial neoplasia: evidence that DPC4 inactivation occurs late in neoplastic progression. Cancer Res. 2000; 60:2002-2006.

11. Biankin AV, Kench JG, Morey AL, Lee CS, Biankin SA, Head DR, Hugh TB, Henshall SM, Sutherland RL. Overexpression of $\mathrm{p} 21$ (WAF1/CIP1) is an early event in the development of pancreatic intraepithelial neoplasia. Cancer Res. 2001; 61:8830-8837.

12. Hahn SA, Schutte M, Hoque AT, Moskaluk CA, da Costa LT, Rozenblum E, Weinstein CL, Fischer A, Yeo CJ, Hruban RH, Kern SE. DPC4, a candidate tumor suppressor gene at human chromosome 18q21.1. Science. 1996; 271:350-353.

13. Yamada S, Fujii T, Shimoyama Y, Kanda M, Nakayama G, Sugimoto H, Koike M, Nomoto S, Fujiwara M, Nakao A, Kodera Y. SMAD4 expression predicts local spread and treatment failure in resected pancreatic cancer. Pancreas. 2015; 44:660-664.

14. Iacobuzio-Donahue $\mathrm{CA}, \mathrm{Fu} \mathrm{B}$, Yachida $\mathrm{S}$, Luo M, Abe H, Henderson CM, Vilardell F, Wang Z, Keller JW, Banerjee P, Herman JM, Cameron JL, Yeo CJ, et al. DPC4 gene status of the primary carcinoma correlates with patterns of failure 
in patients with pancreatic cancer. J Clin Oncol. 2009; 27:1806-1813.

15. Lu D, Reber HA, KraSny RM, Kadell BM, Sayre J. Local staging of pancreatic cancer: criteria for unresectability of major vessels as revealed by pancreatic-phase, thin-section helical CT. AJR American journal of roentgenology. 1997; 168:1439-1443.

16. Lee ES, Lee JM. Imaging diagnosis of pancreatic cancer: A state-of-the-art. World J Gastroenterol. 2014; 20:7864-7877.

17. Bardeesy N, Cheng KH, Berger JH, Chu GC, Pahler J, Olson P, Hezel AF, Horner J, Lauwers GY, Hanahan D, DePinho RA. Smad4 is dispensable for normal pancreas development yet critical in progression and tumor biology of pancreas cancer. Genes Dev. 2006; 20:3130-3146.

18. Blackford A, Serrano OK, Wolfgang CL, Parmigiani G, Jones S, Zhang X, Parsons DW, Lin JC, Leary RJ, Eshleman JR, Goggins M, Jaffee EM, Iacobuzio-Donahue CA, et al. SMAD4 gene mutations are associated with poor prognosis in pancreatic cancer. Clin Cancer Res. 2009; 15:4674-4679.

19. Deckers M, van Dinther M, Buijs J, Que I, Lowik C, van der Pluijm G, ten Dijke P. The tumor suppressor Smad4 is required for transforming growth factor beta-induced epithelial to mesenchymal transition and bone metastasis of breast cancer cells. Cancer Res. 2006; 66:2202-2209.

20. Gal A, Sjoblom T, Fedorova L, Imreh S, Beug H, Moustakas A. Sustained TGF $\beta$ exposure suppresses Smad and nonSmad signalling in mammary epithelial cells, leading to EMT and inhibition of growth arrest and apoptosis. Oncogene. 2008; 27:1218-1230.

21. Zavadil J, Bottinger EP. TGF- $\beta$ and epithelial-tomesenchymal transitions. Oncogene. 2005; 24:5764-5774.

22. Yamada S, Fuchs BC, Fujii T, Shimoyama Y, Sugimoto H, Nomoto S, Takeda S, Tanabe KK, Kodera Y, Nakao A. Epithelial-to-mesenchymal transition predicts prognosis of pancreatic cancer. Surgery. 2013; 154:946-954.

23. Kleeff J, Reiser C, Hinz U, Bachmann J, Debus J, Jaeger D, Friess H, Buchler MW. Surgery for recurrent pancreatic ductal adenocarcinoma. Ann Surg. 2007; 245:566-572.
24. Park JB, Kim YH, Kim J, Chang HM, Kim TW, Kim SC, Kim PN, Han DJ. Radiofrequency ablation of liver metastasis in patients with locally controlled pancreatic ductal adenocarcinoma. J Vasc Interv Radiol. 2012; 23:635-641.

25. Miyazaki M, Yoshitomi H, Shimizu H, Ohtsuka M, Yoshidome H, Furukawa K, Takayasiki T, Kuboki S, Okamura D, Suzuki D, Nakajima M. Repeat pancreatectomy for pancreatic ductal cancer recurrence in the remnant pancreas after initial pancreatectomy: is it worthwhile? Surgery. 2014; 155:58-66.

26. Winter JM, Tang LH, Klimstra DS, Liu W, Linkov I, Brennan MF, D'Angelica MI, DeMatteo RP, Fong Y, Jarnagin WR. Failure patterns in resected pancreas adenocarcinoma: lack of predicted benefit to SMAD4 expression. Ann Surg. 2013; 258:331-335.

27. Boone BA, Sabbaghian S, Zenati M, Marsh JW, Moser AJ, Zureikat AH, Singhi AD, Zeh HJ, Krasinskas AM. Loss of SMAD4 staining in pre-operative cell blocks is associated with distant metastases following pancreaticoduodenectomy with venous resection for pancreatic cancer. J Surg Oncol. 2014; 110:171-175.

28. Saiki Y, Horii A. Molecular pathology of pancreatic cancer. Pathol Int. 2014; 64:10-19.

29. Verbeke C, Leitch D, Menon K, McMahon M, Guillou P, Anthoney A. Redefining the $\mathrm{R} 1$ resection in pancreatic cancer. Br J Surg. 2006; 93:1232-1237.

30. Esposito I, Kleeff J, Bergmann F, Reiser C, Herpel E, Friess H, Schirmacher P, Büchler MW. Most pancreatic cancer resections are R1 resections. Ann Surg Oncol. 2008; 15:1651-1660.

31. Heye T, Zausig N, Klauss M, Singer R, Werner J, Richter GM, Kauczor HU, Grenacher L. CT diagnosis of recurrence after pancreatic cancer: is there a pattern? World J Gastroenterol. 2011; 17:1126-1134. 